\title{
Blood culture sampling practices among internal medicine inpatients
}

\author{
N. Deborah Friedman ${ }^{1 *}$, Tali Braun ${ }^{2}$, Noga Fallach ${ }^{2}$ and Yehuda Carmeli ${ }^{2,3}$ \\ ${ }^{1}$ Departments of Medicine and Infectious Diseases, Barwon Health, Geelong, VIC, Australia \\ ${ }^{2}$ Division of Epidemiology, Tel Aviv Sourasky Medical Center, Tel Aviv, Israel \\ ${ }^{3}$ National Center for Infection Control, Ministry of Health, State of Israel, Israel
}

\begin{abstract}
Objective: Blood cultures are essential for the diagnosis of bloodstream infections; however, they carry a risk of both low yield and culturing skin contaminants. The optimal culturing threshold which results in a high sensitivity of detecting bacteremia yet has a low contamination rate is not well defined. We sought to analyze the blood culture sampling practices, rates and results among patients admitted under internal medicine.
\end{abstract}

Methods: Retrospective analysis of prospectively collected patient data on over 160,000 patient admissions from July 2008 through end of June 2015 at Tel Aviv Sourasky Medical Centre, a 1440-bed tertiary care teaching hospital in Tel Aviv, Israel.

Results: Blood cultures were drawn in $35 \%$ of internal medicine admissions. The overall blood culture sampling rate was 168 blood cultures per 1,000 patient days. $70 \%$ of episodes of suspected bacteremia occurred during the first 48 hours of admission. $4 \%$ of blood cultures yielded a bloodstream infection, while the contamination rate was $3.5 \%$. Single aerobic blood cultures were collected in $35 \%$ of episodes of suspected bacteremia, and the BSI rate among these episodes was $5.3 \%$.

Conclusions: The hospital practice of blood culture sampling varies predominantly by ward and therefore likely by clinician practice. In an internal medicine patient population where the median age was over 70 years the overall blood culture sampling rate was 168 blood cultures per 1,000 patient days and the incidence of BSI was $4 \%$. Single blood cultures are frequently collected, and approximately half of all positive cultures are due to contaminants.

\section{Background}

Blood cultures have an important role in the diagnosis of serious bloodstream infections (BSI) [1], which have an estimated mortality ranging from $14 \%$ to up to $50 \%$, and are a major cause of morbidity and mortality worldwide [2-4]. Blood culture sampling is the most important diagnostic test for bloodstream infections, as it is the only tool that gives reliable information about the causative pathogen and its resistance profile [5].

However, the practice of drawing blood cultures is affected by both the risk of culturing contaminants, and the fact that among adult patients with bacteremia, the yield of blood cultures may be low due to low concentration of organisms (less than 1 organism per $\mathrm{mL}$ of blood) even in the setting of severe sepsis [6,7]. Studies in unselected hospitalized patients have shown that $7.5 \%$ to $12.4 \%$ of all results of blood culture sets are positive; of these, almost half are considered contaminants $[4,8]$. The success of obtaining a positive culture is directly related to the volume of blood that is cultured [9], therefore multiple blood cultures, each containing large volumes of blood, may be required to detect bacteremia [7]. Unfortunately, the Achilles heel of the practice of drawing blood cultures is the costliness and diagnostic uncertainty that results from contaminated or ambiguous blood cultures $[6,7,10]$.

In the 1950s Bennett described bacteremia as usually intermittent [6], and it was this understanding of the transient nature of bacteremia that led to research in the 1970s on the number of blood cultures required to detect bacteremia. Washington demonstrated in cases of bacteremia, that $80 \%$ of cultures are detected as positive in the first set, and that detection increases to $89 \%$ with the second set, and to $99 \%$ with the $3^{\text {rd }}$ set [11]. Expanding on research by Washington, Weinstein and colleagues from the early 1980s shaped the modern clinical practice of drawing blood cultures by finding that $99.6 \%$ of bacteremias were detected by at least 1 of the first 3 blood cultures taken [4]. Similarly, Cockerill found that while 2 blood cultures detected only $80 \%$ of bloodstream infections, 3 blood cultures detected $96 \%$ of episodes [12]. Further research by Weinstein and Reller found that 2 blood cultures obtained during a 24 -hour period will detect $89-93 \%$ of BSIs in adults, while 3 blood cultures over a 24 -hour period will detect all polymicrobial BSIs, and 4 cultures will detect $>99.7 \%$ of unimicrobial BSIs $[3,13]$.

Knowledge about blood culture sampling rates is important for the interpretation of quality indicators such as nosocomial BSIs, however, BSI rates give valid estimates only if a sufficient number of blood cultures are taken [14]. This is however balanced against avoiding performing unwarranted blood cultures when the pretest probability of bacteremia is either low or irrelevant [15-17]. Interventions aimed at raising the standards of blood-culture-taking indications and

Correspondence to: N. Deborah Friedman, Departments of Medicine and Infectious Diseases, Barwon Health, Geelong, VIC, Australia, Tel: (03) 4215 2340; E-mail: deborahf@barwonhealth.org.au

Key words: blood culture, sampling, bacteremia, threshold

Received: January 11, 2017; Accepted: February 03, 2017; Published: February 06,2017 
technique within institutions would likely improve both the quality of patient care and resource use [1].

The Tel Aviv Sourasky Medical Centre is a 1440-bed tertiary care teaching hospital in Tel Aviv, Israel which contains 9 Internal Medicine units geographically located on separate wards with patients admitted to different units in sequential order. The hospital recommendation regarding blood culture collection is that when indicated, 2 sets of aerobic blood cultures should be drawn, with anaerobic cultures reserved for cases where suspicion exists for anaerobic infection. There has however been substantial variation observed in the rate of drawing blood cultures on different wards, assumed to be largely related to individual differences in clinician practice. This study was undertaken to describe and compare the practice of blood culture sampling among inpatients in the different Internal Medicine Units at this institution over a 7 year period, with the aim of determining differences in the rates of blood culture collection and positivity among this patient group.

\section{Methods}

\section{Data collection}

Data were extracted from a hospital computerised database of prospectively collected clinical and microbiological data of adult internal medicine admissions from July 1st 2008 through June 30, 2015. Patients under 18 years of age and those with incomplete data were excluded from the analysis. Data collected included; total number of inpatient admissions and patient days (per month and year), number of blood culture bottles collected, timing of blood culture collection, epidemiology of positive cultures, details of blood culture contaminants. The seasonality of drawing blood cultures was also investigated with an emphasis on any changes in detection of bacteremia during the influenza season of each given year.

\section{Definitions}

An episode of suspected bacteremia was defined as all blood cultures taken within 48 hours of consecutive blood cultures (i.e., any blood culture drawn $\geq 48$ hours after previous blood cultures was considered the indicator of a new episode). We internally validated the use of this definition.

Bacteremia versus contamination: Bacteremia was defined as a recognized pathogen cultured from 1 or more blood cultures. Each positive blood culture at this institution is assessed according to an electronic algorithm based on the number of cultures positive, and the organisms cultured as true-positives, or contaminants [18].

Bloodstream infection (BSI) was defined according to the criteria for laboratory-confirmed BSI proposed by the Centers for Disease Control and Prevention (Atlanta, GA, USA) [19].

Community-acquired (CA) bacteremia was defined as a BSI detected at the time of hospital admission or within the 48 hours after hospital admission. Community-onset (CO) bacteremias included both CA and heath care-associated (HCA) bacteremias (HCA bacteremias were defined as a BSI detected at the time of hospital admission or within 48 hours after hospital admission in a patient with significant exposure to healthcare) [20].

Hospital-acquired bacteremia was defined as BSI detected after hospitalization for 48 hours or longer.

Blood culture sampling rates were defined as the number of blood culture bottles taken divided by the number of patient-days at risk.

\section{Data analysis}

To define an episode of suspected bacteremia an 18-month subset of data was utilized to validate this definition. The exact times that blood cultures were drawn were analyzed as hours from admission and hours from a previous blood culture. The number of blood cultures collected per episode was analyzed, and the number of blood cultures per 1,000 patient days was calculated.

\section{Results}

Admissions: Over the 7-year period from 2008-2015, there were a total of 167,783 admissions to internal medicine. There were 377 admissions of patients under 18 years of age, and 42 admissions with incomplete data, which were excluded leaving a total of 167,364 admissions for analysis, which corresponded to a total of 823,586 hospital days.

Defining an episode of suspected bacteremia: An 18-month subset of the study period (January 2014 to June 2015), was used to examine data and define an episode of suspected bacteremia. During this period there were 33,292 admissions and a total of 35,613 blood culture bottles were collected from 13,231 patient admissions. The majority of blood cultures (72.3\%) were taken within 48 hours of admission to the hospital, and the majority of consecutive blood cultures (85\%) were drawn less than 48 hours apart from each other (Figure 1). Thus, we defined an episode of suspected bacteremia as the period from collection of the first culture until a period of 48 hours without collection of blood cultures.

Blood culture bottles and admissions: In 59,263 patient admissions (35.4\% of all admissions) 138,439 blood culture bottles were drawn. The median number of blood cultures per patient cultured was 2 (IQR 1-3). In 12,529 admissions there was at least 1 positive blood culture and blood culture positivity ranged among medical units from $6.7 \%$ to $8.3 \%$ of admissions (Table 1). In 6,621 patient admissions (4\%) either a pathogen was grown that was considered a typical cause of bacteremia or a skin organism was isolated in more than one blood culture within 48 hours. In the remaining 5,908 patient admissions (3.5\%), blood cultures grew a contaminant (Table 1).

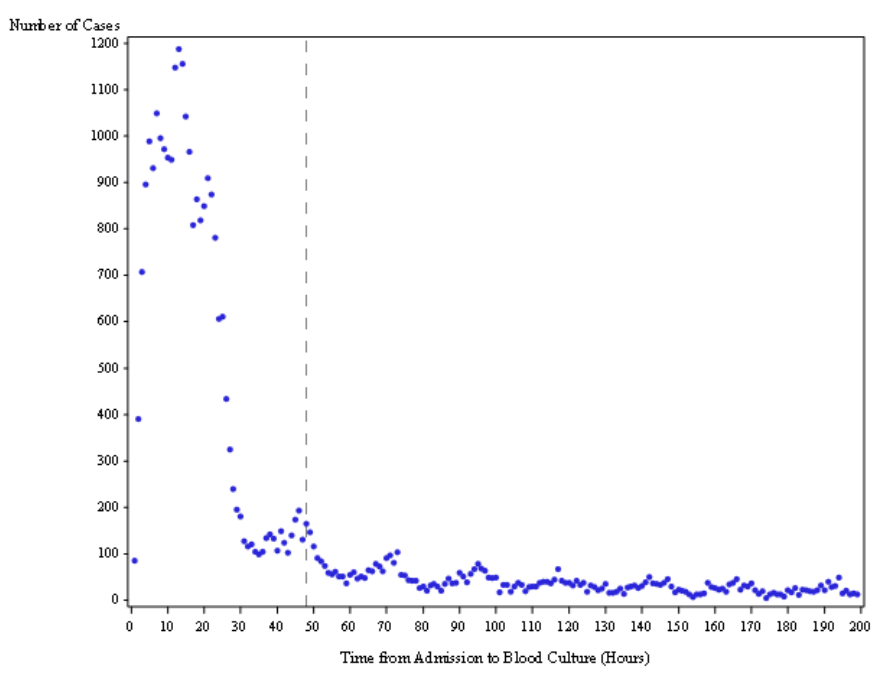

Figure 1. Timing of blood culture collection from hospital admission. 
Table 1. Admissions to Internal medicine, hospital days and blood cultures.

\begin{tabular}{|c|c|c|c|c|c|c|c|c|}
\hline $\begin{array}{l}\text { Internal } \\
\text { Medicine } \\
\text { Unit }\end{array}$ & $\begin{array}{c}\text { Number } \\
\text { of patient } \\
\text { admissions }\end{array}$ & $\begin{array}{c}\text { Total Hospital } \\
\text { Days }\end{array}$ & $\begin{array}{c}\text { Number of patient } \\
\text { admissions with blood } \\
\text { cultures drawn }\end{array}$ & $\begin{array}{c}\text { Total blood } \\
\text { culture bottles } \\
\text { drawn }\end{array}$ & $\begin{array}{l}\text { Blood culture } \\
\text { bottles per } 1,000 \\
\text { patient days }\end{array}$ & $\begin{array}{c}\text { Admissions } \\
\text { with Positive } \\
\text { blood cultures }\end{array}$ & $\begin{array}{l}\text { Admissions with } \\
\text { BSINumber (\%) }\end{array}$ & $\begin{array}{l}\text { Admissions with Positive } \\
\text { Blood cultures with } \\
\text { contaminantsNumbers (\%) }\end{array}$ \\
\hline 1 & 19,716 & 90977 & 6085 & 11,482 & 126.2 & $1329(6.7)$ & $691(3.5)$ & $638(3.2)$ \\
\hline 2 & 19,257 & 87484 & 7108 & 16,192 & 185.1 & $1383(7.1)$ & $721(3.7)$ & $662(3.4)$ \\
\hline 3 & 18,082 & 84366 & 5627 & 13,691 & 162.3 & $1337(7.4)$ & 699 (3.9) & $638(3.5)$ \\
\hline 4 & 18,069 & 99650 & 8377 & 22,910 & 229.9 & $1512(8.3)$ & $784(4.3)$ & $728(4.0)$ \\
\hline 5 & 16,570 & 92292 & 6473 & 16,639 & 180.3 & $1353(8.2)$ & $742(4.5)$ & $611(3.7)$ \\
\hline 6 & 19,097 & 96192 & 5938 & 14,271 & 148.4 & $1422(7.4)$ & $761(4.0)$ & $661(3.4)$ \\
\hline 7 & 19,178 & 96796 & 7105 & 14,845 & 153.4 & $1428(7.4)$ & $781(4.1)$ & $647(3.3)$ \\
\hline 8 & 19,056 & 91324 & 7427 & 17,850 & 195.5 & $1507(7.9)$ & 793 (4.2) & 714 (3.7) \\
\hline 9 & 18,339 & 84505 & 5123 & 10,559 & 125 & $1258(6.8)$ & $649(3.5)$ & $609(3.3)$ \\
\hline Total & 167,364 & 823,586 & $59,263(35.4 \%)$ & 138,439 & 168.1 & $12,529(7.5 \%)$ & $6621(4 \%)$ & $5908(3.5 \%)$ \\
\hline
\end{tabular}

Table 2. Blood culture sampling episodes.

\begin{tabular}{|c|c|c|c|c|c|c|c|c|c|}
\hline $\begin{array}{l}\text { Internal } \\
\text { Medicine } \\
\text { Unit }\end{array}$ & $\begin{array}{l}\text { Number of } \\
\text { episodes }\end{array}$ & $\begin{array}{l}\text { Mean episode } \\
\text { length (max) }\end{array}$ & $\begin{array}{c}\text { Mean number of } \\
\text { BC a bottles per } \\
\text { episode (max) }\end{array}$ & $\begin{array}{l}\text { Episodes per } \\
\text { 1,000 patient } \\
\text { days }\end{array}$ & \begin{tabular}{|c|} 
Episodes with \\
PositiveBC (\%)
\end{tabular} & $\begin{array}{l}\text { Episodes with } \\
\text { BSI (\%) }\end{array}$ & $\begin{array}{c}\text { Episodes with BSI } \\
\text { per } 1,000 \text { patient } \\
\text { days }\end{array}$ & $\begin{array}{c}\text { Episodes with } \\
\text { contaminants } \\
(\%)\end{array}$ & $\begin{array}{c}\text { Episodes with } \\
\text { contaminants per } \\
1,000 \text { patient days }\end{array}$ \\
\hline 1 & 6913 & $1.2(10)$ & $1.7(11)$ & 76 & $1428(20.6)$ & $735.4(10.6)$ & 8.1 & $693(10.0)$ & 7.6 \\
\hline 2 & 8993 & $1.3(13)$ & $1.8(11)$ & 102.8 & $1515(16.8)$ & $771(8.5)$ & 8.8 & $744(8.3)$ & 8.5 \\
\hline 3 & 6604 & $1.3(10)$ & $2.1(12)$ & 78.3 & $1410(21.3)$ & $735(11.1)$ & 8.7 & $675(10.2)$ & 8.0 \\
\hline 4 & 10,424 & $1.3(9)$ & $2.2(12)$ & 104.6 & $1658(15.9)$ & $836(8.0)$ & 8.4 & $822(7.9)$ & 8.3 \\
\hline 5 & 8055 & $1.3(17)$ & $2.1(19)$ & 87.3 & 1493 (18.5) & $802(9.9)$ & 8.7 & $691(8.6)$ & 7.5 \\
\hline 6 & 7075 & $1.2(13)$ & $2.0(29)$ & 73.6 & $1539(21.7)$ & $810(11.4)$ & 8.4 & $729(10.3)$ & 7.6 \\
\hline 7 & 8096 & $1.2(8)$ & $1.8(13)$ & 83.6 & 1499 (18.5) & $823(10.2)$ & 8.5 & $676(8.3)$ & 7.0 \\
\hline 8 & 9222 & $1.3(13)$ & $1.9(13)$ & 101 & 1649 (17.8) & $843(9.1)$ & 9.2 & $806(8.7)$ & 8.8 \\
\hline 9 & 5833 & $1.2(16)$ & $1.8(14)$ & 69 & $1327(22.7)$ & $667(11.4)$ & 7.9 & $660(11.3)$ & 7.8 \\
\hline Total & 71,215 & 1.26 & $2.0(29)$ & 86.5 & $13,518(19)$ & $7022(9.9)$ & 8.5 & $6496(9.1)$ & 7.9 \\
\hline
\end{tabular}

\section{Episodes of suspected bacteremia}

Over the 7 year period, among the 59,263 admissions in which blood cultures were drawn, there were overall 71,215 episodes of suspected bacteremia and 86.5 episodes of suspected bacteremia per 1,000 patient days. There was marked variation in the number of episodes per 1,000 patient days by medical ward (range 69-104.6 episodes) (Table 2).

Episodes had a median duration of 1 day (IQR 1-2 days) and a median of 2 blood culture bottles were collected per episode (IQR 1-2) with a maximum of 29 bottles collected. In 19\% of episodes there was a positive blood culture, ranging between medical units from $15.9 \%$ to $22.7 \%$. 7022 episodes (9.9\% of all episodes of suspected bacteremia) represented BSI, while nearly half of all positive blood cultures yielded contaminants (Table 2).

\section{Community-onset versus Hospital-acquired Episodes of Suspected bacteremia}

55,946 episodes $(78.6 \%)$ occurred during the first 48 hours of admission (referred to as community-onset). BSI was detected in 9.1\% of episodes of community-onset (CO) while contaminants were detected in $8.9 \%$ of CO episodes. 15,269 episodes (21.4\%) were considered hospital-acquired (HA). BSI was detected in $12.5 \%$ of episodes of hospital-onset, while contaminants were detected in $9.8 \%$ of HA episodes.

While half of HA episodes started before or on the 10th day of hospitalization, $85 \%$ of HA episodes started within the first 30 days of hospitalization (Figure 2). The median duration of episodes of suspected bacteremia was 1 day for both $\mathrm{CO}$ and HA episodes. In $\mathrm{CO}$ episodes the median number of blood culture bottles collected was 2 , while for HA episodes the median number of blood culture bottles collected was $1(P<0.01)$. In $31 \%$ of CO episodes 1 blood culture bottle was collected, and in $50 \%$ of episodes 2 bottles were collected. In $50 \%$ of HA episodes 1 blood culture bottle was collected, and in $34 \%$ of episodes 2 bottles were collected

\section{Single blood culture bottles}

In $35 \%$ of episodes of suspected bacteremia $(24,804 / 71,215)$, only a single blood culture bottle was collected (range $21-48 \%$ between different medical wards). Of these 24,804 episodes where single blood culture bottles were collected, 1306 (5.3\%) represented BSI and 1653 (6.7\%) isolated a contaminant. On all medical units, contaminants exceeded BSIs detected from positive single blood culture bottles (Table 3). Single blood cultures were more prevalent in HA episodes of suspected bacteremia.

\section{Discussion}

Since their earliest application, blood cultures have been used whenever physicians suspect a clinically significant bacteremia [17]. The appropriateness of obtaining blood cultures in all patients with suspected infection should however be reevaluated because of the rate of false-positive cultures, the possibility of false-negative cultures, as well as the sometimes limited impact of a true-positive culture on clinical management [21]. In this study there was substantial variation between medical units in the number of episodes of suspected bacteremia. However, the number of BSIs and contaminants detected varied little between medical units, suggesting that the difference in the number of episodes is based on clinician dependent or unit-specific blood culture sampling practices. We also detected both a high rate of negative and false-positive blood culture results among this medical cohort. It is known that there is a low rate of clinically significant truepositive (pathogenic) culture results for common medical inpatient diagnoses such as pneumonia and skin infection [22]. This indicates 


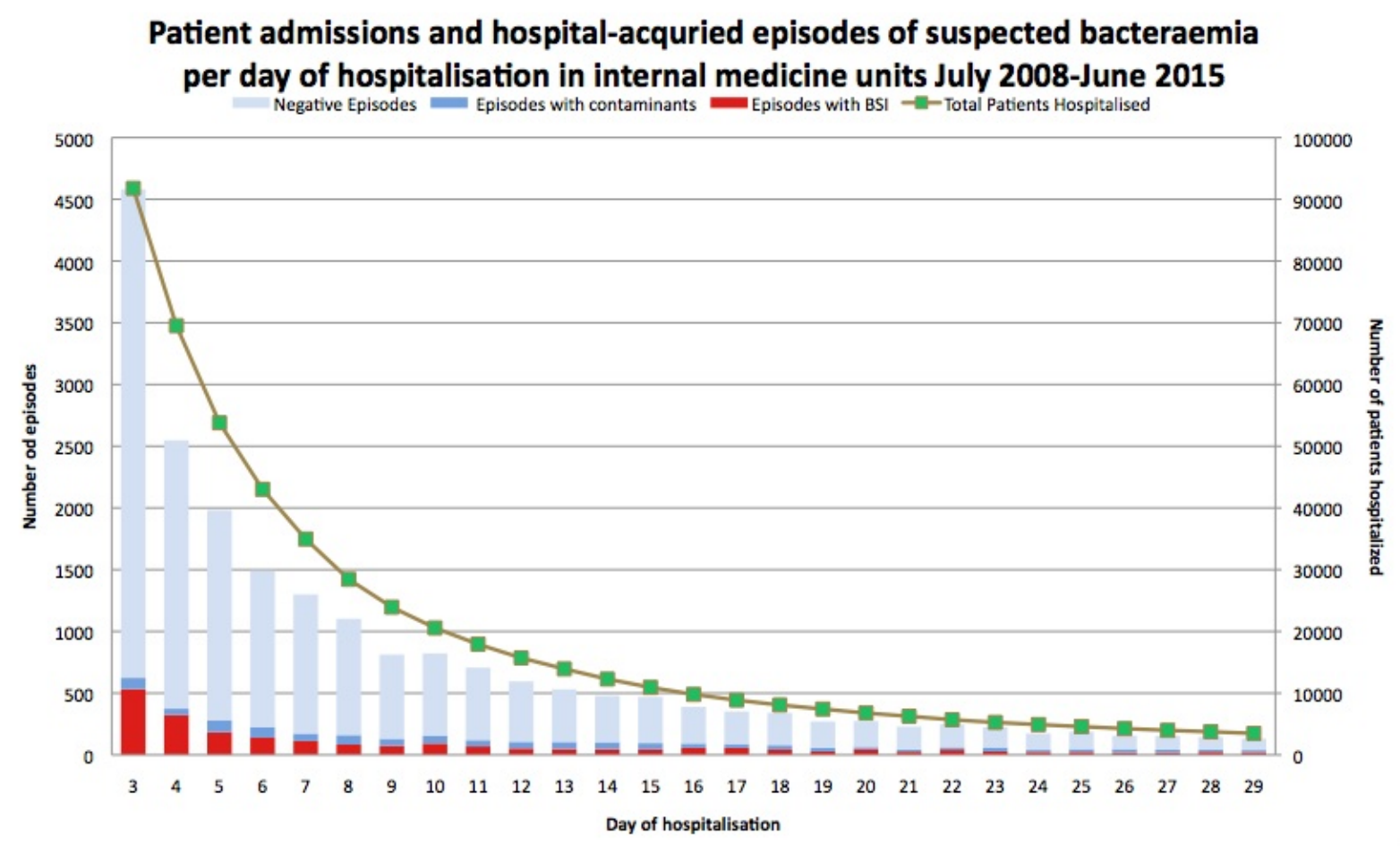

Figure 2. Hospital-acquired episodes of suspected bacteremia by day of hospitalization.

Table 3. Characteristics of episodes with single blood cultures.

\begin{tabular}{|c|c|c|c|c|}
\hline Medical Unit & $\begin{array}{c}\text { Episodes with a single aerobic } \mathrm{BC}^{\text {abottle }} \\
\text { Collected (\%) }\end{array}$ & Positive single BC (\%) & $\begin{array}{l}\text { Bacteremias from single } \\
\text { positive } B C\end{array}$ & $\begin{array}{c}\text { Contaminants from single } \\
\text { positive } B C\end{array}$ \\
\hline 1 & $3358(48.6)$ & $476(14.2)$ & $223(46.9)$ & $253(53.1)$ \\
\hline 2 & $4271(47.5)$ & $459(10.8)$ & $190(41.4)$ & $269(58.6)$ \\
\hline 3 & $1994(30.2)$ & $263(13.2)$ & $106(40.3)$ & $157(59.7)$ \\
\hline 4 & $2274(21.8)$ & $192(8.4)$ & $78(40.6)$ & $114(59.4)$ \\
\hline 5 & $2347(29.1)$ & $240(10.2)$ & $113(47.1)$ & $127(52.9)$ \\
\hline 6 & $1970(27.8)$ & $292(14.8)$ & $135(46.2)$ & $157(53.8)$ \\
\hline 7 & $3262(40.3)$ & $391(12.0)$ & $193(49.4)$ & $198(50.6)$ \\
\hline 8 & 3094 (33.6) & $326(10.5)$ & $138(42.3)$ & $188(57.7)$ \\
\hline 9 & $2234(38.3)$ & $320(14.3)$ & $130(40.6)$ & $190(59.4)$ \\
\hline Total & $24,804(34.8)$ & $2929(12)$ & $1306(44.1)$ & $1653(55.9)$ \\
\hline
\end{tabular}

${ }^{\mathrm{a}} \mathrm{BC}=$ blood culture

that the clinical syndrome of the patient should be a defining factor in influencing whether or not blood cultures should be collected [22].

Much research has been undertaken to better determine when clinicians should order blood cultures in different settings. Leibovici and colleagues created a model based on clinical variables associated with bacteremia, which may improve the clinical diagnosis of patients likely to be bacteremic [23]. Other prediction rules based on clinical features and laboratory abnormalities have been found to be predictive of bacteremia, and could reduce the inappropriate use of blood cultures by approximately $27 \%[21,24]$. It has been suggested that blood cultures should not be ordered for adult patients with isolated fever or leukocytosis without considering the pretest probability [25]. The pretest probability is in turn influenced by the clinical syndrome; where conditions such as cellulitis have a low pretest probability of BSI, and bacterial meningitis would have a high pretest probability of BSI [25].

Contemporary clinical medicine utilizes hospital BSI rates as a key performance indicator. However, reporting observed BSI rates as a quality indicator without sufficiently high blood culture rates might be misleading [14]. From a technical perspective, there is a range in which blood culture rates may be too low for detecting most cases, meaning that a substantial fraction of BSI cases might remain undetected.

In this study of over 160,000 admissions and over 800,000 patient days, we found that in approximately $35 \%$ of internal medicine admissions, blood cultures were drawn which is equivalent to a rate of 168 blood cultures per 1,000 patient-days. Sampling of about 100 to 200 blood culture sets per 1,000 patient-days is recommended as the target range for blood culture rates [26]. However, the empirical justification of this recommendation is not clear. Karch and colleagues studied the blood culture target threshold in 233 German intensive care units [14]. They found that below 80 to 90 blood culture sets per 1,000 patient-days, observed BSI rates increased with increasing blood culture rates, while there was no further increase above this threshold. They located the threshold at 87 blood culture sets (95\% confidence interval, 54 to 120) per 1,000 patient-days [14]. The applicability of this threshold for internal medicine patients who are not in intensive 
care and who have a lower probability of BSI is not known. We suggest that another and perhaps better marker of blood culture sampling may be to analyze the percentage positivity of blood cultures collected, instead of utilizing cultures per patient days. This approach may be easier for microbiology laboratories to utilize and can provide details about contamination rates. In this study, we found that those wards with the lowest blood culture sampling ( 125 blood culture bottles per 1,000 patient days) detected both less true BSIs and less contaminants. In contrast, wards with the highest blood culture sampling rate (229 blood culture bottles per 1,000 patient days) detected both more BSIs and more contaminants.

In this study, we also found that while only a single aerobic blood culture was collected in $35 \%$ of episodes of suspected bacteremia, up to 29 blood culture bottles were collected in some cases. The practice of collecting a single blood culture is contrary to recommendations of collecting 2 to 3 or more blood cultures in cases of suspected bacteremia $[12,13]$. Although single blood cultures have been shown to detect $91 \%$ of BSI episodes [4], they are insufficiently sensitive for detecting some bacteremias and fungemias and they are difficult to interpret [27]. For these reasons, single blood cultures should be discouraged, if not forbidden altogether [17]. It appears that the practice of collecting a single blood culture bottle is widespread in this institution particularly among patients with nosocomial BSI, and the contamination rate among these single blood cultures is high. Unfortunately, changing practices within an institution is difficult as it is likely that clinician practices may be well entrenched from past experiences, and guidelines may have a limited effect on changing physician behavior [28].

In 1996, Weinbaum referred to the contaminant blood culture as a defective product which is associated with increased length of stay, inappropriate administration of antibiotics, and use of further testing, resulting in more than $50 \%$ greater total hospital charges $[7,29]$. According to the American Society of Microbiology, the rate of blood culture contamination should not exceed 3\% [30]. In this study, in nearly 6000 admissions, blood cultures drawn were assessed as contaminants (3.5\% of admissions), which was very similar to the rate of true BSI detected. Interventions targeting both blood culture collection technique and also potentially consideration of dedicated phlebotomists may be appropriate in our setting.

There are limitations to this study. We reviewed blood culture sampling rates, but did not review the individual patient medical records, which would have provided some insight into appropriateness of indications for collecting blood cultures in these cases. We only analyzed aerobic blood culture bottles in this study as the yield from anaerobic cultures is low, and most medical patients do not have anaerobic cultures drawn. It is possible that inclusion of anaerobic cultures may have altered the results. Moreover, we did not address the volume of blood collected in blood cultures, although we acknowledge that this is important in influencing contamination rates [17]. Finally, a description of patient characteristics, the microbiology of blood cultures and patient outcomes is beyond the scope of this study and is studied separately.

Nonetheless, we have ascertained that the hospital practice of blood culture sampling among internal medicine patients does vary predominantly by ward (and therefore likely by clinician practice), that single blood cultures are frequently collected, and that nearly half of all positive cultures are due to contaminants.

\section{Conclusions}

In this study of medical inpatients, the overall blood culture sampling rate was highly variable between wards ranging between 125-229.9 blood cultures per 1,000 patient days (mean 168.1 per 1,000 patient days) with BSI detected in $4 \%$ of admissions, and contaminants detected in $3.5 \%$ of admissions. Of concern, single blood cultures are frequently collected during episodes of suspected bacteremia. Decision rules and guidelines may be helpful in educating clinicians about identifying patients who do or do not need blood cultures and how many cultures to collect.

\section{References}

1. Alahmadi YM, Aldeyab MA, McElnay JC, Scott MG, Darwish FW, et al. (2011) Clinical and economic impact of contaminated blood cultures within the hospital setting. J Hosp Infect 77: 233-236. [Crossref]

2. Martin GS (2012) Sepsis, severe sepsis and septic shock: changes in incidence, pathogens and outcomes. Expert Rev Anti Infect Ther 10: 701-706. [Crossref]

3. Weinstein MP, Towns ML, Quartey SM, Mirrett S, Reimer LG, et al. (1997) The clinica significance of positive blood cultures in the 1990s: a prospective comprehensive evaluation of the microbiology, epidemiology, and outcome of bacteremia and fungemia in adults. Clin Infect Dis 24: 584-602. [Crossref]

4. Weinstein MP, Reller LB, Murphy JR, Lichtenstein KA (1983) The clinical significance of positive blood cultures: a comprehensive analysis of 500 episodes of bacteremia and fungemia in adults. I. Laboratory and epidemiologic observations. Rev Infect Dis 5: 35-53. [Crossref]

5. Hall KK, Lyman JA (2006) Updated review of blood culture contamination. Clin Microbiol Rev 19: 788-802. [Crossref]

6. Doem G (2016) Blood cultures for the detection of bacteremia. In: UpToDate, Rose BD (Ed), UpToDate, Waltham, MA.

7. Bates DW, Goldman L, Lee TH (1991) Contaminant blood cultures and resource utilization. The true consequences of false-positive results. JAMA 265: 365-369. [Crossref]

8. Darby JM, Linden P, Pasculle W, Saul M (1997) Utilization and diagnostic yield of blood cultures in a surgical intensive care unit. Crit Care Med 25: 989-994. [Crossref]

9. Tenney JH, Reller LB, Mirrett S, Wang WL, Weinstein MP (1982) Controlled evaluation of the volume of blood cultured in detection of bacteremia and fungemia. $J$ Clin Microbiol 15: 558-561.

10. Weinstein MP (2003) Blood culture contamination: persisting problems and partial progress. J Clin Microbiol 41: 2275-2278. [Crossref]

11. Washington JA 2nd (1975) Blood cultures: principles and techniques. Mayo Clin Proc 50: 91-98. [Crossref]

12. Cockerill FR III, Wilson JW, Vetter EA, Goodman KM, Torgerson CA, et al. (2004) Optimal testing parameters for blood cultures. Clin Infect Dis 38: 1724-1730. [Crossref]

13. Lee A, Mirrett S, Reller LB, Weinstein MP (2007) Detection of bloodstream infections in adults: how many blood cultures are needed? J Clin Microbiol 45: 3546-3548. [Crossref]

14. Karch A, Castell S, Schwab F, Geffers C, Bongartz H, et al. (2015) Proposing an Empirically Justified Reference Threshold for Blood Culture Sampling Rates in Intensive Care Units. J Clin Microbiol 53: 648-652. [Crossref]

15. Salluzzo R, Reilly K (1991) The rational ordering of blood cultures in the emergency department. Qual Assur Util Rev 6: 28-31. [Crossref]

16. Bates DW, Cook EF, Goldman L, Lee TH (1990) Predicting bacteremia in hospitalized patients. A prospectively validated model. Ann Intern Med 113: 495-500. [Crossref]

17. Aronson MD, Bor DH (1987) Blood cultures. Ann Intern Med 106: 246-253. [Crossref]

18. O'Grady NP, Alexander M, Burns LA, Dellinger EP, Garland J, et al. (2011) Guidelines for the prevention of intravascular catheter-related infections. Clin Infect Dis 52: e162193. [Crossref]

19. Horan TC, Andrus M, Dudeck MA (2008) CDC/NHSN surveillance definition of health care-associated infection and criteria for specific types of infections in the acute care setting. Am J Infect Control 36: 309-332. [Crossref]

20. Friedman ND, Kaye KS, Stout JE, McGarry SA, Trivette SL, et al. (2002) Health careassociated bloodstream infections in adults: a reason to change the accepted definition of community-acquired infections. Ann Intern Med 137: 791-797. [Crossref] 
21. Shapiro NI, Wolfe RE, Wright SB, Moore R, Bates DW (2008) Who needs a blood culture? A prospectively derived and validated prediction rule. J Emerg Med 35: 255264. [Crossref]

22. Parikh K, Davis AB, Pavuluri P (2014) Do we need this blood culture? Hosp Pediatr 4: 78-84. [Crossref]

23. Leibovici L, Greenshtain S, Cohen O, Mor F, Wysenbeek AJ (1991) Bacteremia in febrile patients. A clinical model for diagnosis. Arch Intern Med 151: 1801-1806. [Crossref]

24. Jones GR, Lowes JA (1996) The systemic inflammatory response syndrome as a predictor of bacteraemia and outcome from sepsis. QJM 89: 515-522.

25. Coburn B, Morris AM, Tomlinson G, Detsky AS (2012) Does this adult patient with suspected bacteremia require blood cultures? JAMA 308: 502-511. [Crossref]
26. Seifert H, Abele-Horn M, Fätkenheuer G, Glück T, Jansen B, et al. (2007) Blutkulturdiagnostik: Sepsis, Endokarditis, Katheterinfektionen. In Podbielski A, Hermann M, Kniehl E, Mauch H, Russmann H (ed), Mikrobiologisch-infektiologische Qualitätsstandards (MiQ). Elsevier, München, Germany.

27. Weinstein MP (1996) Current blood culture methods and systems: clinical concepts, technology, and interpretation of results. Clin Infect Dis 23: 40-46. [Crossref]

28. Timmermans S, Mauck A (2005) The promises and pitfalls of evidence-based medicine. Health Aff (Millwood) 24: 18-28. [Crossref]

29. Weinbaum FI, Lavie S, Danek M, Sixsmith D, Heinrich GF, et al. (1997) Doing it right the first time: quality improvement and the contaminant blood culture. J Clin Microbiol 35: 563-565. [Crossref]

30. Reller LB, Murray PR, MacLowry JO (1982) Blood cultures II. In Washington JA II (ed), Cumitech IA. ASM Press, Washington, DC

Copyright: (C2017 N. Deborah Friedman. This is an open-access article distributed under the terms of the Creative Commons Attribution License, which permits unrestricted use, distribution, and reproduction in any medium, provided the original author and source are credited. 